\title{
Acessibilidade e Tecnologia Assistiva no Ambiente Educacional: Mapeamento Sistemático
}

\author{
Gabriella M. T. Alves ${ }^{1}$, Yuska Paola Costa Aguiar ${ }^{1}$ \\ ${ }^{1}$ Universidade Federal da Paraíba (UFPB) - Campus IV - Litoral Norte \\ Rua das Mangueiras, S/N, Companhia de Tecidos Rio Tinto - 58297-000- Rio Tinto - \\ PB - Brasil \\ \{gabi.mayara, yuska\}@dce.ufpb.br
}

\begin{abstract}
Assistive technology consists of equipment, and services, and strategies, and practices designed and implemented to minimize problems encountered by individuals with disabilities. Initiatives in this area should identify the limitations and potential of the subjects in order to establish goals and activities on educational context. This work aims to present the results of a systematic mapping of the literature, whose focus is on understanding how accessibility and assistive technologies have been addressed in educational settings that benefit from computing resources.
\end{abstract}

Resumo. A tecnologia assistiva compreende equipamentos, serviços, estratégias e práticas concebidas e aplicadas para minorar os problemas encontrados pelos indivíduos com deficiências. As iniciativas nessa área devem identificar as limitações e potencialidades desses sujeitos a fim de estabelecer objetivos e atividades no contexto educativo. Este trabalho tem como propósito apresentar o resultado de um mapeamento sistemático da literatura, cujo foco consiste na compreensão sobre como a acessibilidade e as tecnologias assistivas vêm sendo abordadas em ambientes educacionais que usufruem de recursos de informática.

\section{Introdução}

A denominação de "especial" às pessoas que necessitam de atenção diferenciada é um conceito amplo e diversificado. A atual Política Nacional de Educação Especial (POLÍTICA, 2007) aponta para uma definição de prioridades no tocante ao atendimento especializado a ser oferecido na escola para quem dele necessitar. Essa vertente define como aluno portador de necessidades educacionais especiais aquele que "por apresentar necessidades próprias e diferentes dos demais alunos no domínio das aprendizagens curriculares correspondentes à sua idade, requer recursos pedagógicos e metodologias educacionais específicas" (POLÍTICA, 2007).

Os resultados do Censo Demográfico 2010 (IBGE, 2014) apontaram 45.606.048 milhões de pessoas que declararam ter pelo menos uma das deficiências, no qual se baseou na percepção do indivíduo sobre sua dificuldade em enxergar, ouvir ou se locomover, e na existência da deficiência mental ou intelectual, correspondendo a $23,9 \%$ da população brasileira. Logo, classifica-se 18,8\% com deficiência visual, 5,1\% com deficiência auditiva, 7,0\% deficiência motora, 1,4\% deficiência mental ou intelectual e com nenhuma dessas deficiências 76,1\%. Considerando o número significativo, e o pressuposto de que "todos possuírem direitos iguais" se faz necessário propiciar a estas pessoas as mesmas experiências do restante da população, além disso, 
estando incluídas na sociedade em seus diversos níveis, permitindo sua atividade em meios culturais, políticos, educacionais e outros.

Com base nos dados do Censo de 2010 (IBGE, 2014) identificou-se que a taxa de alfabetização das pessoas com mais de 15 anos de idade, sem nenhum tipo de deficiência, foi de $90,6 \%$, no entanto, para a mesma faixa etária essa taxa se reduz para $81,7 \%$ quando se considera o público portador de pelo menos uma deficiência. Passando do nível de alfabetização para a formação no ensino fundamental, 61,1\% das pessoas com pelo menos uma deficiência possuía apenas o fundamental incompleto, no entanto, para pessoas sem nenhum tipo de deficiência o índice foi de 38,2\%. Já com foco no ensino superior incompleto, estão presentes $17,7 \%$ pessoas com deficiência contra 29,7\% para as pessoas sem deficiência. Para o ensino superior completo, apenas $6,7 \%$ pessoas com deficiência e $10,4 \%$ para a população sem deficiência. Nesta análise inicial é possível perceber que existe uma disparidade entre os níveis de formação alcançados pela população deficiente e não deficiente, onde aqueles que são "especiais" encontram dificuldades que os impede de progredir no ambiente educacional oferecido. Ou seja, o setor da educação não esta preparado para receber, manter e formar parte da população, o que se caracteriza um problema social a ser considerado como relevante. Portanto, existe a necessidade de tornar a educação acessível também para estas pessoas. Para Santos (2012) prover a acessibilidade é remover barreiras que impeçam as pessoas com deficiência de participarem de atividades do cotidiano, incluindo o uso de serviços, produtos e informação.

Neste contexto, um dos recursos disponíveis que pode ser utilizado em favor desta demanda é a educação inclusiva que, apoiada pela informática na educação e as tecnologias assistivas, pode auxiliar no processo de ensino-aprendizagem para formação "especial" dos deficientes, respeitando suas capacidades e limitações de forma particular. Diante da importância da inclusão de pessoas com deficiência no âmbito educacional, e meio ao avanço no desenvolvimento de ambiente que promovem esta inclusão. O presente trabalho tem como objetivo fazer um estudo bibliográfico, a partir do mapeamento sistemático da literatura que possibilita uma visão mais ampla dos estudos primários, tornando-o dependente da realização de mapeamentos para revelar as evidências da pesquisa (KITCHENHAM, 2007).

Assim, este artigo apresenta na seção 2 o contexto do trabalho, na seção 3 aborda o método utilizado na pesquisa, na seção 4 são apresentados os resultados e análise e na seção 4 é apresenta a conclusão da pesquisa.

\section{Acessibilidade}

Acessibilidade significa garantir, sobretudo, às pessoas com deficiências ou mobilidade reduzida condições para utilização segura e autônoma de espaços, mobiliários, produtos e informações. De acordo com o Art. $8^{\circ}$ do Decreto $n^{0}$ 5.296/04, são consideradas barreiras na comunicação e informação: "qualquer entrave ou obstáculo que dificulte ou impossibilite a expressão ou o recebimento de mensagens por intermédio dos dispositivos, meios ou sistemas de comunicação, sejam ou não de massa, bem como aqueles que dificultem ou impossibilitem o acesso à informação". Diante disso, a Convenção dos Direitos das Pessoas com Deficiência (BRASIL, 2007), em seu Art. $9^{\circ}$, trata da Acessibilidade que tem por fim possibilitar às pessoas com deficiência viver com autonomia e com plena participação em todos os aspectos de sua vida. No Brasil, 
esta Convenção foi promulgada pelo Decreto $\mathrm{n}^{\circ} 6.949$, de 25 de agosto de 2009 (BRASIL, 2009).

Expandindo o panorama da acessibilidade para os softwares ou ambientes educacionais, sua definição tem gerado discussão no meio científico, em particular em relação ao limiar entre os conceitos de usabilidade e de acessibilidade. Na norma ISO 9241 - Parte 11 (ISO 9241-11), o conceito de usabilidade é definido como: "A medida que um produto (um software ou ambiente) pode ser utilizado por um grupo específico de usuários para atingir objetivos específicos como eficácia, eficiência e satisfação em um contexto específico de uso". Para a mesma norma - Parte 171 (ISO 9241-171) acessibilidade de software é: “... a usabilidade de um produto, serviço, ambiente ou funcionalidade por pessoas com os mais variados tipos de habilidade e capacidades". Nesse contexto, a acessibilidade está relacionada à remoção das barreiras que impedem que mais pessoas possam perceber, compreender e usufruir de todo apoio computacional oferecido pelo ambiente computacional. Dentre essas pessoas estão àqueles portadores de limitações físicas (visuais, auditivas, motoras) e limitações intelectuais, a exemplo dos analfabetos funcionais, aos quais falta domínio nas habilidades de leitura, escrita, cálculos e ciências.

No Brasil, a definição de Tecnologia Assistiva (TA) dada pelo Comitê de Ajuda Técnica (CORDE, 2009) é de uma área do conhecimento, de característica interdisciplinar, que engloba produtos, recursos metodológicos, estratégias, práticas e serviços que objetivam promover a funcionalidade, relacionada à atividade $\mathrm{e}$ participação de pessoas com deficiência, incapacidades ou mobilidade reduzida, visando sua autonomia, independência qualidade de vida e inclusão social. De forma resumida, tecnologia assistiva é "uma ampla gama de equipamentos, serviços, estratégias e práticas concebidas e aplicadas para minorar os problemas encontrados pelos indivíduos com deficiências" (COOK e POLGAR, 1995).

Dentre estas técnicas existem aquelas utilizadas por intermédio de recursos tecnológicos, a fim de possibilitar que pessoas com deficiência interajam com ambientes educacionais. Nesse contexto, a TA se encaixa como aliada enquanto instrumento facilitador de ensino-aprendizagem, oferecendo recursos utilizados para diminuir barreiras, disponibilizar novas formas de interação e comunicação para seus usuários. Considerando as limitações físicas de aprendizes, softwares educacionais podem ser utilizados conjuntamente com recursos que permitam minimizar as dificuldades por estes encontradas, tais como: (i) os softwares leitores de tela, para deficientes visuais, que possuem um sintetizador de voz capaz de obter informações textuais contidas na tela do computador e reproduzi-las em áudio; e (ii) teclado de velocidade reduzida, ponteira de cabeça e software para acompanhamento do movimento dos olhos para usuários com deficiência motora em diferentes níveis de limitações.

Neste meio, nasce o conceito de Educação Inclusiva que pode se apoiar nos recursos de informática disponíveis enquanto TA. Segundo Mrech (2010), a chamada Educação Inclusiva teve início nos Estados Unidos através da Lei Pública 94.142, de 1975 e atualmente, já se encontra na sua segunda década de implementação. Esta busca da escola de qualidade para todos, com a proposta de modificações curriculares com ênfase especial no estabelecimento dos componentes de autodeterminação da criança portadora de deficiência. Na escola inclusiva o processo educativo é entendido como um processo social, onde todas as crianças portadoras de necessidade especiais e de 
distúrbios de aprendizagem têm o direito à escolarização o mais próximo possível do normal, fazendo com que os professores tornem-se mais próximos das crianças para descobrir as suas maiores dificuldades, e assim elas atinjam o seu potencial máximo.

Araújo (2013) destaca que a informática na educação constitui-se de uma área mediadora para facilitar o processo de ensino-aprendizagem nos mais diversos campos do conhecimento. Valente (1991) defende que na vertente da educação inclusiva são desenvolvidas as TAs para atender as demandas específicas de pessoas com necessidades especiais no âmbito sócio-educacional. $\mathrm{O}$ uso da informática aliado à constante renovação de equipamentos e softwares especialmente desenvolvidos para atender necessidades especiais faz do computador um instrumento importante para inserção dos deficientes em ambientes ricos de aprendizagem, contribuindo para que tenhamos uma sociedade de participação plena e de igualdade para todos os seus indivíduos, princípios básicos da inclusão.

\section{Método de Pesquisa}

Este trabalho se caracteriza como um estudo teórico-conceitual, uma vez que busca investigar as atuais abordagens adotadas para promover acessibilidade nos ambientes educacionais, visando ainda identificar as características compatíveis entre as diferentes abordagens, assim como lacunas existentes neste contexto de estudo. Para tanto, executou-se um mapeamento sistemático de literatura para o levantamento de trabalhos sobre ambientes educacionais sob o aspecto de acessibilidade. Segundo Kitchenham et al. (2007) e Brereton et al. (2007) uma revisão sistemática deve ser realizada seguindo as etapas de planejamento, condução e comparativo, podendo estas serem utilizadas para mapeamento sistemático. As sessões seguintes detalham as etapas realizadas e seu emprego para a realização deste trabalho de pesquisa.

\subsection{Planejamento}

Esta etapa consiste em definir um conjunto de questão de pesquisa que devem ser utilizadas como critérios específicos para filtrar os trabalhos a serem considerados no estudo e direcionar a busca ao objetivo definido. As questões de pesquisa devem ser respondidas ao final do mapeamento. Em adicional, define-se um conjunto de critérios de inclusão e exclusão, filtros que, de acordo com Ramos (2014), têm o objetivo de identificar estudos primários e que sejam relevantes para a pesquisa. Nesta etapa definese ainda os motores busca e as palavras-chaves (strings) a serem utilizadas e o idioma base para a pesquisa.

O Quadro 1 apresenta as decisões consideradas no planejamento da pesquisa. A escolha pelas bibliotecas digitais utilizadas foi motivada por estas serem internacionalmente conhecidas no âmbito acadêmico e pela qualidade dos estudos indexados, além do acesso fácil e rápido aos autores. Como consequência, as strings apresentam-se na língua inglesa. 


\section{Quadro 1: Planejamento da revisão sistemática de literatura}

\section{Questões de Pesquisa}

Quais são as abordagens de inclusão adotadas para promover acessibilidade no ambiente educacional?

Quais as áreas do conhecimento que o ambiente educacional abrange?

A qual público (cegos, surdos, motores, intelectuais) a acessibilidade é direcionada?

\section{Critérios de inclusão}

Incluir na pesquisa estudos realizados nos últimos 4 anos.

\section{Critérios de Exclusão}

Trabalhos que apenas citam os ambientes virtuais educacionais, mas não apresentam uma metodologia para sua utilização;

Estudos duplicados (foi considerada a versão mais completa de artigos existentes);

Short papers com menos de três páginas;

White papers, relatórios técnicos e livros;

Estudos secundários: revisões da literatura, mapeamentos, entre outros;

$\begin{array}{ll}\text { Motores de busca } & \text { Palavras-chave (Strings de busca) } \\ \text { ACM Digital } & \text { ((Accessibility in educational environments) OR (Evaluation of Virtual } \\ \text { Library } & \text { Environments) OR (Accessibility) AND (Educational Environments)). } \\ \text { IEEE Xplore } & \begin{array}{l}\text { ("accessibility" OR "deficiency") AND ("evaluation" OR "virtual } \\ \text { Digital Library }\end{array} \\ \begin{array}{l}\text { environments" OR "educational environments"). } \\ \text { ((Accessibility in educational environments) OR (Accessibility to virtual } \\ \text { environments) AND (evaluation Educational Environments) OR } \\ \text { (education)). }\end{array}\end{array}$

\subsection{Condução}

A condução do mapeamento realizou-se de acordo com o protocolo detalhado na seção 3.1, a saber: execução da string de busca nos motores de busca, aplicação dos critérios de inclusão e exclusão, seguida da leitura dos trabalhos resultantes para identificar quais deles respondem as questões de pesquisa, resultando no cospus considerado para a análise.

A execução da string de busca formatada para cada base de dados resultou na localização de 858 registros, sendo reduzidos a um universo de 62 registros após a aplicação dos critérios de inclusão e exclusão. Na busca por respostas as questões de pesquisa, os registros foram analisados através da leitura inicial do resumo, da introdução, da proposta apresentada, e da conclusão deste. Como resultado, houve redução no número de registros de interesse, finalizando em um universo de apenas 11 trabalhos (Tabela 1).

Tabela 1: Quantidade de trabalhos antes de adotar a metodologia utilizada.

\begin{tabular}{|l|c|c|}
\multirow{2}{*}{ Motores de busca } & \multicolumn{2}{c|}{ Quantidade de registros } \\
\cline { 2 - 3 } Busca inicial & Resultado final \\
\hline ACM Digital Library & 446 & 8 \\
\hline IEEE Xplore Digital Library & 60 & 0 \\
\hline Scopus & 352 & 3 \\
\hline Total & $\mathbf{8 5 8}$ & $\mathbf{1 1}$ \\
\hline
\end{tabular}

\footnotetext{
${ }^{1}$ ACM Digital Library. Disponível em: http://dl.acm.org/

${ }^{2}$ IEEE Xplore Digital Library. Disponível em: http://ieeexplore.ieee.org/Xplore/home.jsp

${ }^{3}$ Scopus. Disponível em: http://www.scopus.com/
} 


\subsection{Comparativo}

Considerando o universo de registros relevantes para responder as questões de pesquisa definidas, tem-se o ponto de partida para analisar e refletir acerca das atuais abordagens utilizadas no contexto da acessibilidade para ambientes educacionais, considerando os desafios enfrentados, as metodologias utilizadas e o próprio ambiente educacional resultante de cada estudo considerado. No Quadro 2, tem-se a lista dos títulos considerados, onde cada referência está associada a um identificador único. Os trabalhos estão apresentados em ordem decrescente quanto ao ano de publicação, do mais recente para o mais antigo.

\section{Quadro 2: Lista dos títulos considerados na pesquisa}

\begin{tabular}{|c|c|c|}
\hline Ano & ID & Títulos considerados \\
\hline 2014 & 1 & $\begin{array}{l}\text { PEREZ-GONZALEZ, Daniel; SOTO-ACOSTA, Pedro; POPA, Simona. A Virtual Campus } \\
\text { for E-learning Inclusion: The Case of SVC-G9. J. UCS, v. 20, n. 2, p. 240-253, } 2014 .\end{array}$ \\
\hline \multirow{3}{*}{2013} & 2 & $\begin{array}{l}\text { SOGONO, Maria Carmela; RICHARDS, Deborah. A design template for multisensory and } \\
\text { multimodal games to train and test children for sound localisation acuity. In: Proceedings } \\
\text { of The 9th Australasian Conference on Interactive Entertainment: Matters of Life and } \\
\text { Death. ACM, 2013. p. 25. }\end{array}$ \\
\hline & 3 & $\begin{array}{l}\text { VICKERS, Stephen; ISTANCE, Howell; HYRSKYKARI, Aulikki. Performing } \\
\text { Locomotion Tasks in Immersive Computer Games with an Adapted Eye-Tracking } \\
\text { Interface. ACM Transactions on Accessible Computing (TACCESS), v. 5, n. 1, p. 2, } 2013 .\end{array}$ \\
\hline & 4 & $\begin{array}{l}\text { QUEK, Francis; OLIVEIRA, Francisco. Enabling the blind to see gestures.ACM } \\
\text { Transactions on Computer-Human Interaction (TOCHI), v. 20, n. 1, p. } 4,2013 \text {. }\end{array}$ \\
\hline \multirow{3}{*}{2012} & 5 & $\begin{array}{l}\text { HAILPERN, Joshua et al. Designing visualizations to facilitate multisyllabic speech with } \\
\text { children with autism and speech delays. In: Proceedings of the Designing Interactive } \\
\text { Systems Conference. ACM, 2012. p. 126-135. }\end{array}$ \\
\hline & 6 & $\begin{array}{l}\text { SMITH, Kel. Universal life: the use of virtual worlds among people with } \\
\text { disabilities. Universal Access in the Information Society, v. 11, n. 4, p. 387-398, } 2012 \text {. }\end{array}$ \\
\hline & 7 & $\begin{array}{l}\text { SANTAROSA, Lucila Maria Costi; CONFORTO, Débora; BASSO, Lourenço de Oliveira. } \\
\text { Eduquito: ferramentas de autoria e de colaboração acessíveis na perspectiva da web } 2.0 \text {; } \\
\text { Eduquito: accessible authorship and collaboration tools from the perspective of web } \\
\text { 2.0. Rev. bras. educ. espec, v. } 18, \text { n. } 3 \text {, p. } 449-468,2012 \text {. }\end{array}$ \\
\hline \multirow{2}{*}{2011} & 8 & $\begin{array}{l}\text { WYETH, Peta; SUMMERVILLE, Jennifer; ADKINS, Barbara. Stomp: an interactive } \\
\text { platform for people with intellectual disabilities. In: Proceedings of the 8th International } \\
\text { Conference on Advances in Computer Entertainment Technology. ACM, 2011. p. } 51 \text {. }\end{array}$ \\
\hline & 9 & $\begin{array}{l}\text { LUDI, Stephanie; REICHLMAYR, Tom. The use of robotics to promote computing to pre- } \\
\text { college students with visual impairments. ACM Transactions on Computing Education } \\
\text { (TOCE), v. } 11, \text { n. } 3 \text {, p. } 20,2011 \text {. }\end{array}$ \\
\hline \multirow[b]{2}{*}{2010} & 10 & $\begin{array}{l}\text { SÁNCHEZ, Jaime; SÁENZ, Mauricio; GARRIDO, Jose Miguel. Usability of a multimodal } \\
\text { video game to improve navigation skills for blind children. ACM Transactions on } \\
\text { Accessible Computing (TACCESS), v. 3, n. } 2 \text {, p. } 7,2010 \text {. }\end{array}$ \\
\hline & 11 & $\begin{array}{l}\text { KUSHALNAGAR, Raja S.; CAVENDER, Anna C.; PÂRIS, Jehan-François. Multiple } \\
\text { view perspectives: improving inclusiveness and video compression in mainstream } \\
\text { classroom recordings. In: Proceedings of the 12th international ACM SIGACCESS } \\
\text { conference on Computers and accessibility. ACM, 2010. p. 123-130. }\end{array}$ \\
\hline
\end{tabular}

Buscou-se destacar, para cada título, os pontos interessantes e sua contribuição diante do contexto abordado (Quadro 3).

Em princípio é possível identificar uma variedade de objetivos abordados por estas pesquisas, assim como dos recursos utilizados e deficiências a serem assistidas. $\mathrm{Na}$ seção seguinte estes trabalhos são analisados em função de responder as questões de pesquisas definidas no planejamento desta pesquisa bibliográfica. 


\section{Quadro 3: Características dos trabalhos}

\begin{tabular}{|c|c|c|c|c|}
\hline ID & Software & Objetivo & Recursos e Técnicas & $\begin{array}{l}\text { Deficiências } \\
\text { Alvo }\end{array}$ \\
\hline 1 & $\begin{array}{l}\text { Learning } \\
\text { Management } \\
\text { Systems }\end{array}$ & $\begin{array}{l}\text { Distribuição de cursos, } \\
\text { gerenciamento de conteúdo } \\
\text { e comunicação. }\end{array}$ & $\begin{array}{l}\text { Leitor de tela, mouse, } \\
\text { teclado, emulador de teclado. }\end{array}$ & $\begin{array}{l}\text { Cognitiva, } \\
\text { Visual, Motora, } \\
\text { Auditiva e } \\
\text { Neurológica. }\end{array}$ \\
\hline 2 & Jogo eletrônico & $\begin{array}{l}\text { Coerência, informação } \\
\text { verbal, personalização das } \\
\text { telas, voz e sinalização, e } \\
\text { interatividade. }\end{array}$ & $\begin{array}{l}\text { Método estruturado para } \\
\text { descrever o jogo e ajudar na } \\
\text { sua utilização para o } \\
\text { ambiente educacional. }\end{array}$ & Auditiva \\
\hline 3 & Jogos online & $\begin{array}{c}\text { Independência dos jogadores } \\
\text { no posicionamento e } \\
\text { direcionamento dos } \\
\text { personagens. }\end{array}$ & $\begin{array}{l}\text { Técnica "olho no olho". } \\
\text { Utilização de ambientes } \\
\text { imersivos para fins } \\
\text { educacionais. }\end{array}$ & Motora \\
\hline 4 & $\begin{array}{l}\text { Haptic Deictic } \\
\text { System }\end{array}$ & $\begin{array}{c}\text { Aprendizagem em salas de } \\
\text { aula inclusivas. }\end{array}$ & $\begin{array}{l}\text { Interface tátil sobre o gráfico } \\
\text { em conjunto com a fala. }\end{array}$ & Visual \\
\hline 5 & VocSyl & $\begin{array}{l}\text { Interação social, e } \\
\text { relacionamento de propósito } \\
\text { educacional. }\end{array}$ & $\begin{array}{c}\text { Sistema de voz para a } \\
\text { visualização em tempo real. }\end{array}$ & Neurológica \\
\hline 6 & Second Life & $\begin{array}{l}\text { Autonomia presente nos } \\
\text { mundos virtuais permita que } \\
\text { os usuários superem a } \\
\text { autopercepção que podem } \\
\text { limitar a sua capacidade. }\end{array}$ & Realidade aumentada. & $\begin{array}{l}\text { Motora e } \\
\text { Neurológica }\end{array}$ \\
\hline 7 & Eduquito & $\begin{array}{l}\text { Colocar o aprendiz no centro } \\
\text { do processo educativo. }\end{array}$ & $\begin{array}{l}\text { Lista de atalhos via teclado; } \\
\text { aumento/ redução do } \\
\text { tamanho da fonte; texto de } \\
\text { ajuda sensível ao contexto; } \\
\text { vídeo em LIBRAS; áudio } \\
\text { que reproduz texto. }\end{array}$ & $\begin{array}{l}\text { Cognitiva, } \\
\text { Visual, Motora, } \\
\text { Auditiva e } \\
\text { Neurológica. }\end{array}$ \\
\hline 8 & Stomp & $\begin{array}{l}\text { Criar experiência acessível e } \\
\text { inclusiva através das } \\
\text { interações encorpada } \\
\text { habilitada por uma interface } \\
\text { tangível. }\end{array}$ & $\begin{array}{l}\text { Tapete com sensores de } \\
\text { pressão embutidos. }\end{array}$ & Intelectual \\
\hline 9 & $\begin{array}{l}\text { Oficinas } \\
\text { educativas }\end{array}$ & $\begin{array}{l}\text { Oficinas para envolver } \\
\text { alunos com programação } \\
\text { robótica através dos kits } \\
\text { Legam Mindstorm NXT. }\end{array}$ & $\begin{array}{c}\text { Ferramenta de programação } \\
\text { para robótica e material } \\
\text { didático projetado. }\end{array}$ & Visual \\
\hline 10 & TheMOVA3D & $\begin{array}{l}\text { Estimular as habilidades de } \\
\text { orientação tempo-espacial } \\
\text { em crianças. }\end{array}$ & Áudio. & Visual \\
\hline 11 & $\begin{array}{l}\text { Multiple View } \\
\text { Perspectives. }\end{array}$ & $\begin{array}{c}\text { Escolher o foco da } \\
\text { aprendizagem em sala de } \\
\text { aula. }\end{array}$ & $\begin{array}{l}\text { Conjunto de vídeo, com foco } \\
\text { em cada região da sala de } \\
\text { aula. }\end{array}$ & Auditiva \\
\hline
\end{tabular}

\section{Respondendo as questões de pesquisa}

Como caracterização geral do corpus analisado, a partir dos 11 artigos incluídos percebe-se um crescimento no número de publicações entre os anos de 2010 e 2014, consequência do critério de inclusão adotado. No entanto, é possível que algum artigo publicado neste período não tenha sido analisado. Outra informação secundária, resultante deste levantamento, consiste na identificação dos países origem das publicações, onde têm-se a distribuição: 4 trabalhos nos Estados Unidos, 2 na Austrália, 2 no Brasil, e os demais se dividem entre Chile, Espanha e Reino Unido. 
Questão de Pesquisa 1: Quais são as abordagens de inclusão adotadas para promover acessibilidade no ambiente educacional? Destacam-se: 6 trabalhos com abordagem voltada a proposta de solução utilizando softwares/ferramentas $[1,4,5,8,10$ e 11]; 2 trabalhos que utilizam jogos eletrônicos como abordagem para superar a auto percepção e capacidade dos usuários [2 e 6]; 1 trabalho utiliza a técnica "olho no olho", onde os usuários ao utilizar os jogos online, têm a sensação de dependência ao realizar tarefa [3]; 1 trabalho sobre desenvolvimento de um ambiente virtual de aprendizagem [7]; e, por fim, a aplicação de oficinas para envolver alunos com a programação robótica [9].

Questão de Pesquisa 2: Quais as áreas do conhecimento que o ambiente educacional abrange? Foi possível observar que os trabalhos não se limitam a expor áreas específicas de conhecimento (matemática, biologia, computação, etc.), mas sim um conjunto delas. $\mathrm{O}$ objetivo geral está direcionado para favorecer a interação das pessoas com deficiência e em ambientes comuns de atividades realizadas no dia-a-dia, havendo foco na integração social, na autoestima e na sua formação educacional.

Questão de Pesquisa 3: A qual público (cegos, surdos, motores, intelectuais) a acessibilidade é direcionada? Constatou-se que 2 artigos [1 e 7] são abrangentes em relação a conjunto de deficiências para qual se destina, a saber, cognitiva, visual, motora, auditiva ou neurológica; 1 com ênfase na deficiência motora ou neurológica [6]; 2 para deficiência auditiva [2 e 11]; 3 para deficiência visual [4, 9 e 10], e os outros 3 se dividem em deficiência motora [3], neurológica [5] e intelectual [8].

\subsection{Análise dos resultados}

$\mathrm{Na}$ análise destacam-se os ambientes educacionais direcionados aos deficientes visuais (5 dos 11 trabalhos), esta predominância indica uma preocupação com a formação deste público, motivada, talvez, pela elevada concentração da população acometida por esta limitação (incluindo os cegos e aqueles de baixa-visão). A abrangência nas áreas de conhecimento abordadas nos trabalhos pode ser interpretada como uma necessidade latente de inserção social dos deficientes que se faz presente através dos ambientes educacionais. Não existe um direcionamento destas tecnologias para disciplinas específicas de conhecimento, como matemática, biologia, ciências, pela necessidade, urgente, de tornar este público independente em atividades cotidianas e que envolvem o ambiente educacional. O impacto destas ações reflete não apenas no processo de formação cognitiva e intelectual, mas proporciona o autoconhecimento no reconhecimento de suas capacidades e possibilidades de superar limitações, gerando motivação para aqueles que usufruem destes recursos. Embora os trabalhos analisados façam menção aos recursos e tecnologias assistivas utilizadas para promover acessibilidade em ambientes educacionais, não existe clareza sobre os procedimentos metodológicos utilizados para o desenvolvimento de tais ambientes.

Portanto, não é possível avaliar a adequação destes as necessidades pedagógicas e de acessibilidade a serem consideradas em um ambiente de aprendizagem para pessoas com necessidades especiais.

\section{Considerações Finais}

Neste trabalho apresenta-se o resultado da realização de um mapeamento sistemático da literatura cujo objetivo consistiu em identificar como a questão da acessibilidade e das tecnologias assistivas estão sendo abordadas em ambientes educacionais que usufruem 
de recursos de informática. A revisão se apoiou em três motores de busca reconhecidos academicamente em âmbito internacional (ACM Digital Library, IEEE Xplore Digital Library e Scopus) e utilizou-se de um conjunto de strings de busca na língua inglesa. As publicações consideradas para análise, após a aplicação dos critérios de inclusão e exclusão, consistem em 11 artigos publicados entre 2010 e 2014.

Mesmo considerando um número restrito de publicações, é possível identificar a preocupação com o tema de pesquisa em países da América, Europa e Oceania, levando à compreensão de que se trata de uma problemática que não é localizada no Brasil. Além disto, considerando as questões de pesquisa, foi possível identificar (i) uma leve predominância de soluções propostas para portadores de deficiência visual; (ii) uma abrangência nas áreas de conhecimento abordadas, cujo foco está direcionado para atividades cotidianas no ambiente educacional; e (iii) ausência de descrição sobre os procedimentos metodológicos utilizados no desenvolvimento das soluções apresentadas.

Embora cientes dos resultados alcançados, percebe-se como principal limitação deste trabalho de pesquisa o pequeno número de artigos contemplados no estudo, devido a redução de itens a serem considerados após a aplicação dos critérios de inclusão e exclusão (de 858 para 62) agravada após a busca pelas respostas para as questões da pesquisa dentro deste universo de registros (redução de 62 para 11). Sendo uma importante questão de pesquisa (1) a investigação sobre as abordagens de inclusão adotadas para promover acessibilidade no ambiente educacional, a ausência desta informação nos artigos restringiu a formação do corpus considerado. Esta limitação pode ser resultante da área de conhecimento investigada (carente), de problemas na execução do processo de revisão de literatura (derivada da realização de análise manual), ou ainda da possível inserção de erros em alguma das etapas de execução do protocolo de pesquisa (escolha dos critérios, strings de busca ou motores de busca). A limitação referente ao tamanho do corpus, trabalhos que adote uma abordagem metodológica para promover acessibilidade ao ambiente educacional, pode ser compreendida como um indicador da necessidade de melhor direcionar a atenção da sociedade e comunidade acadêmica para o desenvolvimento de soluções acessíveis, adequadas e mediadoras no processo de ensino-aprendizagem para pessoas com deficiência.

O desafio no processo de ensino-aprendizagem é fazer com que uma turma heterogenia, em termos de deficiência, seja compreendida enquanto unidade (grupo) ao utilizar uma mesma plataforma para realização de atividades de ensino-aprendizagem, levando ao sentimento de homogeneidade das partes. Logo, como trabalho futuro, sugere-se o desenvolvimento de um software adaptativo à diferentes deficiências (públicos) a partir da união de vários softwares em um único ambiente de aprendizagem. Pretende-se, contemplar recursos adequados à necessidade de cada aluno para a realização de atividades de ensino-aprendizagem, trabalhando para a inclusão em sala de aula e reforçando a importância da socialização entre os envolvidos.

\section{Referências}

Araújo, A. L. S. O.; Brito, R. R.; Silva, A. P. (2013) "Softwares para educação inclusiva: uma revisão sistemática no contexto de SBIE e WIE", In: II Congresso Brasileiro de Informática na Educação e XXIV Simpósio Brasileiro de Informática na Educação, Campinas, SP. 
Brasil. Convenção sobre os direitos das pessoas com deficiência. Brasília: SEDH/CORDE, 2007. Disponível em: <http://portal.mec.gov.br/> Acesso em: 20 jun. 2014.

Brasil. Decreto $\mathrm{n}^{\mathrm{o}}$ 6.949, de 25 de agosto de 2009. "Promulga a Convenção Internacional sobre os Direitos das Pessoas com Deficiência e seu Protocolo Facultativo, assinados em Nova York, em 30 de março de 2007”. Diário Oficial [da] República Federativa do Brasil, Brasília, DF, 26 ago. 2009. Seção 1, p. 3.

Brereton, P., Kitchenham, B. A., Budgen, D., Turner, M., Khalil, M. (2007) "Lessons from applying the systematic literature review process within the software engineering domain”, In: Journal of Systems and Software 80.

Brasil. Subsecretaria Nacional de Promoção dos Direitos da Pessoa com Deficiência. Comitê de Ajudas Técnicas. Tecnologia Assistiva - Brasília: CORDE, 2009. 138 p.

Cook, A. M; Polgar, J. M. (1995) "Assistive Technologies: Principles and Practices", In: Mosby - Year Book, Inc.

IBGE - Instituto Brasileiro de Geografia e Estatística (2012). "Censo demográfico 2010: Características gerais da população, religião e pessoas com deficiência”. Rio de Janeiro, RJ.

ISO 9241-11 (1998) “International Standards Organization 9241-11. 1998. Ergonomic requirements for office work with visual display terminals (VDTs) - Parte 11: guidance on usability".

ISO 9241-171 (2008) "International Standards Organization 9241-171. 2008. Ergonomics of human-system interaction - Parte 171: Guidance on software accessibility".

Mrech, L. M. (2010) “O que é Educação Inclusiva?” Disponível em: http://www.luzimarteixeira.com.br/wp-content/uploads/2010/10/1-o-que-e-educacaoinclusiva.pdf $>$. Acesso em: 20 jul. 2014.

Kitchenham, B.; Charters, S. (2007) "Guidelines for performing systematic literature reviews in software engineering", In: Technical Report EBSE 2007 - 001, Keele University and Durham University Joint Report.

Política Nacional de Educação Especial na Perspectiva da Educação Inclusiva. "Documento elaborado pelo Grupo de Trabalho nomeado pela Portaria Ministerial $\mathrm{n}^{\circ}$ 555, de 5 de junho de 2007”. Disponível em: http://portal.mec.gov.br/arquivos/pdf/politicaeducespecial.pdf. Acesso em: 20 jul. 2014.

Ramos, F. G. (2014) "Reusabilidade em SOA: Um Mapeamento Sistemático da Literatura" In: Dissertação do Curso de Bacharelado em Sistemas de Informação do Centro de Ciências Aplicadas e Educação (CCAE), Campus IV da Universidade Federal da Paraíba, Rio Tinto, PB.

Santos L. G. et al. (2012) "Recursos de Acessibilidade para Auxiliar a Navegação de Estudantes Cegos em um Editor de Diagramas", In: 23 Simpósio Brasileiro de Informática na Educação (SBIE 2012), Rio de Janeiro, Novembro de 2012.

Valente, J. A. (1991) "Liberando a Mente: Computadores da Educação Especial”, In: Campinas: Editora da UNICAMP. 